\title{
Nuclear Fusion Induced by Super-Intense Ultrashort Laser Pulses in Deuterium Cluster Plasma
}

\author{
V.P. Krainov ${ }^{a}$ and M.B. Smirnov \\ ${ }^{a}$ Moscow Institute of Physics and Technology \\ 141700 Dolgoprudny, Moscow Region, Russia \\ ${ }^{b}$ Max-Born Institute, Rudower Chaussee 6, 12489 Berlin, Germany \\ An $\mathrm{SiO}_{2}$ aerogel with absorbed deuterium is suggested as a target for \\ the fusion reaction $\mathrm{d}+\mathrm{d} \rightarrow \mathrm{He}^{3}+\mathrm{n}$ by a super-intense ultrashort laser pulse. \\ The multiple inner ionization of oxygen and silicon atoms in the fibers of \\ this fractal aerogel takes place in super-intense laser field. The Coulomb \\ explosion of the deuterated aerogel skeleton propels deuterons up to ki- \\ netic energies of ten $\mathrm{keV}$ and higher. The neutron yield is estimated up \\ to $10^{5}$ neutrons per laser pulse during $\approx 500 \mathrm{ps}$ if the peak intensity is \\ $10^{18} \mathrm{~W} / \mathrm{cm}^{2}$ and pulse duration is $35 \mathrm{fs}$.
}

PACS numbers: 52.57.Bc, 52.57.Kk, 28.52.Cx

\section{Introduction}

A new laser technique with the generation of femtosecond super-intense pulses is a basis of new methods for the generation of neutrons. In this way some schemes have been suggested [1-5] with usage of table-top lasers. The approach of Ditmire et al. [1-4] used the fusion reaction between two deuterium nuclei (deuterons) which proceeds according to the classical scheme $\mathrm{d}+\mathrm{d} \rightarrow \mathrm{He}^{3}+\mathrm{n}$. In this method large clusters of deuterium molecules are irradiated by a super-intense ultrashort laser pulse, and explosion of the produced positively charged clusters consisting only of deuterons leads to the formation of a plasma whose electrons and deuterons have the energy of several $\mathrm{keV}$ each. The fusion reaction between deuterons proceeds after the explosion of charged clusters, but before the plasma total decay out of the laser focal volume. Schwoerer et al. [5] demonstrated a 
two-step scheme of neutron generation. On the first stage X-ray photons are emitted by a target irradiated by a super-intense laser pulse, and, on the second stage, $\mathrm{X}$-ray photons create neutrons as a result of interaction with the nucleus $\mathrm{Be}^{9}$.

The neutron yield had been observed up to $n_{\mathrm{n}} \approx 10^{4}$ neutrons per laser pulse in experiments $[3,4]$ at the peak laser intensity of $I \approx 10^{17} \mathrm{~W} / \mathrm{cm}^{2}$ and the pulse width of $35 \mathrm{fs}$. In the laser focusing region with the focal spot of $2 r=$ $200 \mu \mathrm{m}$ and the length of $l=2 \mathrm{~mm}$ [4] the laser focal volume is $V=\pi r^{2} l \approx$ $6.3 \times 10^{-5} \mathrm{~cm}^{3}$; the experimental number density of deuterons has been found to be equal to $N_{\mathrm{d}} \approx(2 \div 3) \times 10^{19} \mathrm{~cm}^{-3}$. Hence, the total number of deuterons in this plasma filament is of order of $n_{\mathrm{d}}=N_{\mathrm{d}} V \approx 10^{15}$. Thus, only one of the $n_{\mathrm{d}} / n_{\mathrm{n}}=10^{11}$ deuterons takes part in the nuclear fusion reaction! This extremely low efficiency is explained by a small tunneling rate of the fusion reaction and by a small lifetime of the forming plasma involving deuteron filament $(\approx 200 \div 500 \mathrm{ps})$.

The neutron yield can be increased by an increase in a kinetic energy of deuterons which is small compared to the classical threshold energy $180 \mathrm{keV}$ of the fusion reaction involving two deuterons. In turn, the cluster expansion leads to formation of fast deuterons under the action of the positive electric potential of the deuteron cluster ion. There is an optimal cluster size at given parameters of the laser pulse. Indeed, a small cluster size leads to a small electric potential and, respectively, to a small energy of deuterons in a plasma, whereas the laser signal cannot ionize fully a large deuterium cluster. In particular, under conditions of the experiments $[3,4]$ the typical cluster radius is approximately $R \approx 25 \AA$ that corresponds to the cluster charge $\approx+3000 e$ after field removal of all electrons, and provides a typical kinetic energy of deuterons of several keV [6] (though the fusion reactions are produced mainly from small amount of clusters with the radii of $50-80 \AA)$.

\section{Aerogel properties}

One can increase a typical deuteron kinetic energy by an increase in the laser intensity. We now suggest an alternative approach when an aerogel with absorbed deuterium is used as a target for laser irradiation, instead of the deuterium cluster beam. The character of processes with formation of fast deuterons is similar in both cases, but, in our opinion, the aerogel method can provide a higher deuteron kinetic energy. We analyze in this paper the processes resulting from irradiation of a typical $\mathrm{SiO}_{2}$ aerogel with absorbed deuterium by an ultrashort super-intense laser pulse.

An aerogel can be described within the frames of a simple model when the aerogel matter consists of bound solid cylindrical fibers of an identical radius a which have common knots. These fibers form a stable rigid skeleton due to rigid contacts between the neighboring fibers. At small distances from a fiber an aerogel has a fractal structure with the fractal dimensionality $D$. An aerogel matter is a 
homogeneous structure starting from a distance $R_{c}$ which is called the correlation radius [7]. An aerogel presents a fractal matter at distances $r<R_{\mathrm{c}}$. These quantities are the basic aerogel parameters.

The radius $a$ of the individual fiber is connected with the specific internal aerogel surface $S$ (which is measured usually in $\mathrm{m}^{2} / \mathrm{g}$ ) by the known relation [7]:

$$
S=\frac{2}{a \rho_{0}},
$$

where $\rho_{0}$ is the (solid) mass density of the aerogel fibers. Another relation between aerogel parameters which follows from its fractal structure, is of the form [7]:

$$
\frac{\rho}{\rho_{0}}=\left(\frac{a}{R_{c}}\right)^{3-D},
$$

where $\rho$ is the average aerogel (gas) mass density. For definiteness, we use below the typical $\mathrm{SiO}_{2}$ aerogel parameters: $S=450 \mathrm{~m}^{2} / \mathrm{g}, \rho=0.005 \mathrm{~g} / \mathrm{cm}^{3}, D=2.3$. Since the solid density of the $\mathrm{SiO}_{2}$-aerogel material is $\rho_{0}=2.2 \mathrm{~g} / \mathrm{cm}^{3}$, we obtain from Eq. (1) that $a=20 \AA$, and from Eq. (2) that $R_{\mathrm{c}}=12.0 \mu \mathrm{m} \ll r=100 \mu \mathrm{m}$.

The $\mathrm{SiO}_{2}$ molecule mass is $m_{0}=1.0 \times 10^{-22} \mathrm{~g}$. The number density of $\mathrm{SiO}_{2}$ molecules in the fiber is $N_{0}=\rho_{0} / m_{0}=2.2 \times 10^{22} \mathrm{~cm}^{-3}$. The mass of the aerogel in the laser focal volume $V$ is $M=\rho V=3.1 \times 10^{-7} \mathrm{~g}$.

Let us note that the maximum amount of absorbed deuterium matter corresponds, obviously, to its solid mass density $\rho_{\mathrm{d}}=0.17 \mathrm{~g} / \mathrm{cm}^{3}$ inside the aerogel, since pores possess a main part of the aerogel volume. Hence, the maximum amount of absorbed deuterium is $\rho_{\mathrm{d}} / \rho=34 \mathrm{~g}_{\mathrm{d}} / \mathrm{g}_{\mathrm{a}}$ (gram of deuterium matter per aerogel gram). However, the optimal amount of absorbed deuterium should be, of course, much less. We suppose that an internal aerogel surface $S$ can be covered by three deuterium layers. This assumption is in agreement with laws of surface physical chemistry [8]. Deuterons are attracted to the free ends of the oxygen radicals. Since the average distance between deuterium molecules in solid deuterium matter is $3.5 \AA$, then the thickness of deuterium matter on the surface of the aerogel is approximately equal to $l_{\mathrm{d}}=10.5 \AA$. Then the amount of absorbed deuterium is only $\rho_{\mathrm{d}} l_{\mathrm{d}} S=0.080 \mathrm{~g}_{\mathrm{d}} / \mathrm{g}_{\mathrm{a}}$. Thus, the laser radiation is mainly absorbed by $\mathrm{SiO}_{2}$ aerogel instead of deuterium.

The total length $L$ of the fibers in the plasma filament with the volume $V$ is found from the obvious relation $\rho_{0} \pi a^{2} L=\rho V$. Thus, we obtain $L=147 \mathrm{~km}$. Let us estimate now the total length $l_{c}$ of the all fibers inside the correlation sphere with the radius $R_{c}$. Obviously, it can be found from the relation $l_{c}=\left(4 \pi R_{c}{ }^{3} / 3 V\right) L$. Substituting the above obtained values of $V, L$, and $R_{\mathrm{c}}$, we find $l_{\mathrm{c}}=16.9 \mathrm{~m}$.

Now let us estimate the average distance $\delta \gg a$ between the neighboring fibers in the correlation sphere. The average length of the one fiber in this sphere is the size of the sphere, i.e. $\sim R_{\mathrm{C}}$. The quantity $\sim \delta^{2} R_{\mathrm{C}}$ is the volume referring to the one fiber in this sphere. The quantity $N_{\mathrm{f}} \sim R_{\mathrm{c}}^{3} / \delta^{2} R_{\mathrm{c}}$ is the number of fibers in this sphere. Hence, $l_{\mathrm{c}} \sim R_{\mathrm{c}} N_{\mathrm{f}}$. Thus, we find $\delta \sim R_{\mathrm{c}} \sqrt{R_{\mathrm{c}} / l_{\mathrm{c}}} \approx 0.01 \mu \mathrm{m}$. 


\section{An aerogel in a laser field}

As a result of irradiation of an aerogel by a super-intense ultrashort laser pulse the following processes proceed. Through some time electrons are liberated from the aerogel skeleton by the laser field and occupy aerogel pores. Then the aerogel skeleton consists of multicharged atomic ions of silicon and oxygen; deuterium nuclei (deuterons) are located on the internal skeleton surface. On the next evolution stage all the atomic ions fly into a surrounding space forming a uniform plasma which fills all the aerogel space; deuterons fly the first because they are the light particles. Deuterons have the maximum kinetic energy since they are located on the skeleton surface.

A general character of processes of interaction of a power laser pulse with the aerogel system is similar to that in the case of deuterium clusters. Hence, below we use the estimations which were done by us for explosion of deuterium clusters under the action of a super-intense ultrashort laser pulse [6].

We assume that the laser peak intensity is $I_{\max }=10^{18} \mathrm{~W} / \mathrm{cm}^{2}$, laser wavelength is $\lambda=800 \mathrm{~nm}$ and the pulse width is $\tau=35 \mathrm{fs}$ (defined as full width at a half maximum, see [9]). Then the laser field strength $F(t)$ is defined as

$$
F(t)=F_{\max } \exp \left[-(t / 30)^{2}\right] \quad(t \text { in fs })
$$

and the peak laser field strength is $F_{\max }=5.25$ a.u. (the linear polarization of the laser field is considered).

Now let us consider in detail the processes which take place during interaction of laser light with an aerogel. First the multiple inner ionization of silicon, oxygen and deuterium atoms occurs in this laser field (of course, the molecular bonds are destroyed very quickly). Taking into account the known values of the ionization potentials $E_{Z}$ of multicharged atomic ions of $\mathrm{Si}$ and $\mathrm{O}(Z$ is the charge multiplicity of the corresponding atomic ion) we find that the above-barrier inner multiple field ionization occurs in the laser field when the Bethe condition is satisfied [10] (here and throughout we use the system of atomic units $e=m_{\mathrm{e}}=\hbar=1$ ):

$$
F(t)>\frac{E_{Z}^{2}}{4 Z} .
$$

Using the known values of the ionization potentials of atomic ions [11] we find from Eq. (3.1) that at the leading edge of the laser pulse the charge multiplicity is $Z_{\mathrm{O}}=6$ for oxygen atomic ions. Thus, each oxygen atomic ion conserves only two electrons of $K$-shell since $K$-shell ionization potentials are very high: $739 \mathrm{eV}$ and $871 \mathrm{eV}$, respectively. Further, $Z=9$ for silicon atomic ions, i.e. each $\mathrm{Si}$ atomic ion conserves only two electrons of $K$-shell and three electrons of $L$-shell. The ionization potential of the $\mathrm{Si}$ atomic ion with $Z_{\mathrm{Si}}=9$ is equal to $401 \mathrm{eV}$, and this quantity does not already satisfy the condition (3.1) even at $F(t)=F_{\max }$. We neglect the quantum-mechanical tunneling atomic ionization in the beginning of the laser pulse since its probability is too small compared to the probability of 
the classical above-barrier ionization [12]. Thus, we find that $Z=2 \times 6+9=$ 21 electrons leave each $\mathrm{SiO}_{2}$ molecule during the inner ionization. After inner ionization the (atomic) unit of the fiber length contains $Z_{\mathrm{f}}=Z N_{0} \pi a^{2} \approx 310$ free electrons.

Simultaneously the outer above-barrier ionization proceeds during the laser pulse, i.e. the electrons leave the aerogel fiber, and we can calculate the number of electrons $Q$ removed from the fiber (per atomic unit of its length) by the laser field. According to the Gauss electrostatic theorem the electric field strength $F_{\mathrm{C}}(r)$ on the distance of $r$ from the fiber axis is found from the relation $2 \pi r F_{\mathrm{C}}(r)=4 \pi Q$. The electric potential is $\varphi(r)=2 Q \ln (a / r)$. The field strength $F_{0}$ on the surface of the fiber is equal to $F_{Q}=2 Q / a$. Electrons continue to leave the fiber under the condition $F(t)>2 Q / a$. Hence,

$$
Q=F_{\max } a / 2 \approx 100 .
$$

The thermal mechanism of outer ionization for femtosecond range of time and for moderate dimensions of individual aerogel fibers gives an additional, but small contribution (see estimates in the review paper [13] and discussion below). Thus, one third of the all free electrons is removed from the fiber by the laser field so that after laser pulse the charge of atomic unit of the fiber is equal to $Q \approx 100$. Further, after Coulomb explosion of the fibers all $n_{\mathrm{t}}=Z_{\mathrm{f}} L=7.4 \times 10^{17}$ free electrons will present in the volume $V$ of plasma filament.

Free electrons are heated during a laser pulse. An electron cannot acquire the average oscillation energy $F^{2} / 4 \omega^{2}$ from the laser field since the amplitude of its oscillations $F / \omega^{2}$ is much greater than the fiber size $a$. The kinetic energy of an electron is of order of its ionization potential (tens of $\mathrm{eV}$ ). Thus, free electrons are remaining quite cold immediately after the inner ionization. But then free electrons can increase their kinetic energy significantly in the process of the fiber explosion. Coulomb potential energy of electrons inside the fiber is transformed into their kinetic energy [6]. As a result, the electron temperature increases up to the final value of $T_{\mathrm{e}} \approx 1 \mathrm{keV}$. Besides of this, the induced inverse bremsstrahlung [14, 15] in the plasma filament can contribute into the increase in the electron kinetic energy when electrons are scattered by the charged fibers before the laser pulse depletes. The amount of this contribution is unclear due to quick explosion of these fibers.

Let us note that the Coulomb expansion of an individual fiber proceeds slowly in comparison with the laser pulse duration. Indeed, the time $t$ for doubling of the fiber radius can be estimated from the simple energy balance for the silicon atomic ion (or oxygen atomic ion) at the fiber surface (see analogous estimates in Ref. [15] for the case of spherical clusters)

$$
2 Q \ln 2=\frac{1}{2} M_{0} v^{2}=\frac{1}{2} M_{0}\left(\frac{2 a}{t}\right)^{2} .
$$

Here $M_{0}$ is the mass of the single silicon atomic ion, and $v$ is its velocity for the 
time moment $t$. It follows from this relation that the fiber radius $a$ is doubled during the time

$$
t=a \sqrt{\frac{M_{0}}{Q \ln 2}} \approx 20 \mathrm{fs} .
$$

Hence, we can neglect the expansion of the individual fiber before the total ejection of all $Q=100$ electrons from the atomic unit of the fiber length during the laser pulse. Estimates for the oxygen atomic ion result in the analogous conclusion.

\section{Penetration of the strong laser field in plasma}

An electromagnetic field inside plasma is screened by means of displacement of plasma electrons; a typical distance for this screening is the Debye radius

$$
r_{\mathrm{D}}=\sqrt{\frac{T_{e}}{4 \pi N_{e}}} .
$$

In particular, for parameters of the cluster plasma under consideration, where the electron number density $N_{\mathrm{e}}=Z_{\mathrm{f}} L / V \approx 2.0 \times 10^{-3}$ a.u. and $T_{\mathrm{e}} \approx 1 \mathrm{keV}$, we obtain $r_{\mathrm{D}} \approx 70 \AA$. A weak electromagnetic wave cannot propagate into a density plasma when the frequency of Langmuir oscillations $\omega_{\mathrm{p}}=\sqrt{4 \pi N_{e}}$ for this plasma exceeds the electromagnetic wave frequency $\omega$. In the case under consideration we have $\omega_{\mathrm{p}}=4.26 \mathrm{eV}$; this quantity is greater than the laser frequency $\omega=1.55 \mathrm{eV}$.

The criterion of a weakness of the electric field is of the form

$$
F r_{\mathrm{D}} \ll T_{\mathrm{e}} .
$$

In particular, in the considered case we find $F \ll F_{\text {cr }}=T_{\mathrm{e}} / r_{\mathrm{D}}=0.26$ a.u., while the peak intensity of the electromagnetic wave $I_{\max }=10^{18} \mathrm{~W} / \mathrm{cm}^{2}\left(F_{\max }=5.25 \mathrm{a.u}\right.$. $)$ corresponds to the opposite inequality.

Displacements of the plasma electrons create an additional electric field which shields the incident electromagnetic field. The screening of the external field occurs in a plasma region near its boundary, and the width of this region is of the order of the Debye radius $r_{\mathrm{D}}$. This region contains a restricted number of plasma electrons; therefore it cannot shield strong laser fields.

First we analyze the penetration of a constant longitudinal electric field into an equilibrium classical plasma. Let us consider the one-dimensional Poisson equation for the electric potential $\varphi(x)$ :

$$
\frac{\mathrm{d}^{2} \varphi(x)}{\mathrm{d} x^{2}}=4 \pi N_{\mathrm{e}}\left[\exp \left(\frac{\varphi(x)}{T_{\mathrm{e}}}\right)-1\right],
$$

where $N_{\mathrm{e}}$ is the average number density of electrons or ions, and the plasma boundary is chosen at $x=0$. A plasma is located in a half-space $x \geq 0$.

In the case of a weak electric field we can linearize Eq. (4.3), and its well known solution is of the form (for $x \geq 0$ ): 


$$
\varphi(x)=\varphi_{0} \exp \left(-x / r_{\mathrm{D}}\right), \quad F(x)=F_{\max } \exp \left(-x / r_{\mathrm{D}}\right), \quad F_{\max }=\varphi_{0} / r_{\mathrm{D}} .
$$

Here the Debye radius $r_{\mathrm{D}}=\sqrt{T_{\mathrm{e}} / 4 \pi N_{\mathrm{e}}}$ determines the shielding of a weak electric field in a plasma. The quantity $F_{\max }$ is the field strength at $x=0$. The boundary conditions are $\varphi(0)=\varphi_{0}$, and $\varphi(\infty)=0$. This solution is valid under the condition (4.2) of a weak electric field $\left|\varphi_{0}\right|=F_{\max } r_{\mathrm{D}} \ll T_{e}$ that allows us to linearize Eq. (4.3).

Let us consider now the opposite limiting case of a strong electric field $\left|\varphi_{0}\right| \gg T_{e}$. Here $\varphi_{0}=\varphi(0)$. If $\varphi_{0}<0$ (and $F_{\max }<0$ ) we can neglect the first term $\exp \left[\varphi(x) / T_{\mathrm{e}}\right]$ in the right side of Eq. (4.3). Then the solution of the simple differential equation

$$
\frac{\mathrm{d}^{2} \varphi(x)}{\mathrm{d} x^{2}}=-4 \pi N_{\mathrm{e}}
$$

can be written in the form

$$
\begin{aligned}
& \varphi(x)=\varphi_{0}+\left|F_{\text {max }}\right| x-2 \pi N_{e} x^{2}<0, \quad F(x)=-\left|F_{\text {max }}\right|+4 \pi N_{e} x<0, \\
& 0<x<x_{0}=\left|F_{\text {max }}\right| / 4 \pi N_{e}, \quad \varphi_{0}=-\left|F_{\text {max }}\right| x_{0} / 2 .
\end{aligned}
$$

Thus, the quantity

$$
x_{0}=\frac{\left|F_{\max }\right|}{4 \pi N_{\mathrm{e}}} \gg r_{\mathrm{D}}
$$

is the screening radius for the case of a strong electric field. This solution corresponds to the repulsion of electrons from the boundary of plasma $(x=0)$. Numerical solution of Eq. (4.3) confirms these analytical results. The estimate of $x_{0}$ for $F_{\max }=5.25 \mathrm{a} . \mathrm{u}$. and for the above cited value of $N_{\mathrm{e}}=2.0 \times 10^{-3} \mathrm{a} . \mathrm{u}$. is $x_{0} \approx 115 \AA$.

Thus, we can conclude that if the electric field is variable, then during a half of a period this field penetrates well in a plasma. During the second part of the laser period the penetration diminishes strongly. Further, though the laser radiation is a transverse electromagnetic wave, we can consider the arbitrary direction of the wave propagation through the aerogel matter and apply again Eq. (4.4).

The laser energy is absorbed by free electrons in the plasma filament. According to above consideration each electron acquires the energy $E_{\mathrm{e}} \approx 10-100 \mathrm{eV}$ from the laser field (other part of the electron energy $\approx 1 \mathrm{keV}$ is acquired from the Coulomb potential energy of the electrons in the fiber). If the laser energy in the pulse is, for example, $E=1 \mathrm{~J}=6.2 \times 10^{18} \mathrm{eV}$, then we find that $n_{1}=E / E_{\mathrm{e}} \approx$ $10^{17}$ electrons absorb the whole energy of the laser pulse. But we have found above that the total number of free electrons in the plasma filament is $n_{\mathrm{t}}=Z_{\mathrm{f}} L=7.4 \times 10^{17}$ that is nearly to $n_{\mathrm{l}}$. Thus, we conclude that the high-intensity laser pulse can be totally absorbed by free electrons of the aerogel.

This analysis leads to the following conclusions. First, a standard concept that the electromagnetic wave with the frequency $\omega<\omega_{\mathrm{p}}$ cannot propagate in a plasma (see, for example, [16]) is not valid at high intensity of the electromag- 
netic field when the criterion (4.2) is violated. Second, the propagation of a strong electromagnetic wave causes the redistribution of plasma charges, and interaction of the electromagnetic wave with a forming non-uniform plasma may be of importance for plasma heating. Note that above we neglected this interaction, and formation of the plasma under the action of the electromagnetic wave results only in separation of electrons and ions of the aerogel skeleton by the wave. A partial absorption of an electromagnetic wave by a forming plasma as a result of collective excitations in this plasma may contribute an additional heating of the plasma. Deep penetration of ultrahigh-intensity laser pulse into a dense plasma is confirmed also by theoretical results obtained in Ref. [17].

\section{Fusion reactions}

Finally, let us estimate the number of fusion neutrons which are produced in the plasma after the end of the laser pulse. During the preliminary diffusion of the deuterium gas through the aerogel the deuterium molecules penetrate inside the pores of aerogel; then they stick to the surface of the fibers. It should be noted that, probably, it is better to use a heavy water, instead of a deuterium gas, because the $\mathrm{D}_{2} \mathrm{O}$ molecule has a dipole moment and attaches to the skeleton surface better than the nonpolar $\mathrm{D}_{2}$ molecule. Besides of this, the oxygen atom adds 6 free electrons for each $\mathrm{D}_{2} \mathrm{O}$ molecule at the irradiation by laser pulse.

Now we consider the Coulomb explosion of an aerogel in the model of the cylindrical fibers. After the inner and outer ionization the atomic unit of length of the fiber contains the electric charge $Q=100$. The difference of electric potentials between the surface of the fiber and the neighboring fiber is $\Delta \varphi=2 Q \ln (\delta / a)=$ $27 \mathrm{keV}$. This quantity is equal to the maximum kinetic energy of the deuterium nucleus. We use a half of this value as the average deuteron kinetic energy.

The cross-section of the fusion reaction $\mathrm{d}+\mathrm{d} \rightarrow{ }^{3} \mathrm{He}+\mathrm{n}$ for the deuteron kinetic energy $E_{\mathrm{d}}=14 \mathrm{keV}$ is $\sigma=5 \times 10^{-29} \mathrm{~cm}^{2}$ [18] (the reaction $\mathrm{d}+\mathrm{d} \rightarrow$ $\mathrm{t}+\mathrm{p}$ has the same cross-section, but we are not interested in this reaction channel here).

If the quantity $S=450 \mathrm{~m}^{2} / \mathrm{g}$ is the specific area of aerogel, and $M=\rho V$ is the mass of the aerogel $\left(\rho=0.005 \mathrm{~g} / \mathrm{cm}^{3}\right.$ is the mass density of the aerogel and $V$ is the volume of the plasma filament), then the quantity $M S$ is the total area of aerogel in the plasma filament. This area is covered by three layers of deuterium molecules. The thickness of this layer $l_{\mathrm{d}}=10.5 \AA$. Hence, the quantity $M S l_{\mathrm{d}}$ is the volume of the deuterium layer, and the quantity $\rho_{\mathrm{d}} M S l_{\mathrm{d}}$ is its mass ( $\rho_{\mathrm{d}}=0.17 \mathrm{~g} / \mathrm{cm}^{3}$ is the mass density of the solid deuterium matter). The number of deuterium molecules in the plasma filament is $n_{\mathrm{d}}=\rho_{\mathrm{d}} M S l_{\mathrm{d}} / M_{\mathrm{d}}\left(M_{\mathrm{d}}=\right.$ $6.7 \times 10^{-24} \mathrm{~g}$ is the mass of the one deuterium molecule). Thus, the number density of deuterons in the plasma filament is $N_{\mathrm{d}}=n_{\mathrm{d}} / V=\rho_{\mathrm{d}} \rho S l_{\mathrm{d}} / M_{\mathrm{d}}=6 \times 10^{19} \mathrm{~cm}^{-3}$. This value is comparable to the corresponding value in the case of deuterium clusters [4]. 
The rate for this nuclear reaction is $w=\sigma N_{\mathrm{d}} v$ where $v$ is the deuteron velocity. The time for nuclear fusion is determined by the time flight $T$ of the deuteron from the axis of the laser focal volume to its radial boundary, i.e. $T=r / v$. Hence, each deuteron produces the number $w T=\sigma N_{\mathrm{d}} r=3 \times 10^{-11}$ neutrons at the collisions with other deuterons. The total yield of neutrons $n_{\mathrm{n}}$ per laser pulse can be obtained multiplying this quantity by the number $n_{\mathrm{d}}$ of deuteron pairs in the laser focal volume: $n_{\mathrm{n}}=w T n_{\mathrm{d}} \approx 10^{5}$.

\section{Conclusion}

We can conclude that the yield of neutrons is greater approximately ten times compared to the yield of neutrons at the irradiation of deuterium clusters by super-intense ultrashort laser pulse observed in the experiments [1-4] (see also recent theoretical calculations for deuterated clusters in Ref. [19]). Thus, an aerogel saturated by deuterium can be used for production of powerful source of ultrashort pulses (about of $T=200 \div 500 \mathrm{ps}$ ) of monochromatic neutrons $(2.45 \mathrm{MeV}$ ).

Developing the method by Zweiback et al. [3, 4] for production of neutrons under the action of a super-intense ultrashort laser pulse, we suggest to use the aerogel skeleton with absorbed deuterium instead of deuterium cluster in the Zweiback et al. experiments. This allows us to increase the neutron yield per laser pulse by one order of magnitude because of a higher kinetic energy of deuterons which are liberated at the Coulomb explosion of the charged aerogel skeleton. It should be noted that the interesting theoretical approach had been suggested recently in Ref. [20]: to use heteronuclear clusters containing deuterium, for example, clusters from $\mathrm{D}_{2} \mathrm{O}$ molecules.

It is possible that the tungsten aerogel considered in Ref. [21] has an advantage compared to the $\mathrm{SiO}_{2}$ aerogel considered in our paper, due to high charge of the tungsten nucleus. Note in conclusion that the Lawson criterion is $\approx 10^{-5}$ in the case of deuterium clusters and $\approx 10^{-4}-10^{-3}$ in the aerogel case. The suggested method can be discussed as a version for the basis of the laser thermonuclear reactor.

\section{Acknowledgments}

This work was supported by the Russian Foundation for Basic Research (projects N 01-02-16056 and N 01-02-06514) and by Civil Research and Development Foundation (grant MO-011-0). Authors are deeply grateful to V.E. Fortov, T. Ditmire, P. Parks, and I.Yu. Skobelev for useful discussions. 


\section{References}

[1] T. Ditmire, J. Zweiback, V.P. Yanovsky, T.E. Cowan, G. Hays, K.B. Warton, Nature (London) 398, 489 (1999).

[2] J. Zweiback, R.A. Smith, V.P. Yanovsky, T.E. Cowan, G. Hays, K.B. Warton, T. Ditmire, in: Multiphoton Processes ICOMP VIII, Eds. L.F. DiMauro, R.R. Freeman, K.C. Kulander, AIP, New York 2000, p. 534.

[3] J. Zweiback, R.A. Smith, T.E. Cowan, G. Hays, K.B. Warton, V.P. Yanovsky, T. Ditmire, Phys. Rev. Lett. 84, 2634 (2000).

[4] J. Zweiback, T.E. Cowan, R.A. Smith, J.H. Hartley, R. Howell, C.A. Steinke, G. Hays, K.B. Warton, J.K. Crane, T. Ditmire, Phys. Rev. Lett. 85, 3640 (2000).

[5] H. Schwoerer, H. Gibbon, S. Düsterer, R. Behrens, C. Ziener, C. Reich, R. Sauerbrey, Phys. Rev. Lett. 86, 2317 (2001).

[6] V.P. Krainov, M.B. Smirnov, Zh. Eksp. Teor. Fiz. 119, 719 (2001) [Phys. JETP 92, $626(2001)]$.

[7] B.M. Sminnov, Phys. Rep. 188, 1 (1990); Physics of Fractal Clusters, Nauka, Moscow 1993.

[8] K. Christmann, Introduction to Surface Physical Chemistry, Springer, New York 1991.

[9] T. Ditmire, Phys. Rev. A 57, R4094 (1998).

[10] H. Bethe, E.E. Salpeter, Quantum Mechanics of One- and Two Electrons Atoms, 2nd ed., Rosetta, New York 1977.

[11] Handbook of Chemistry and Physics, Ed. D.R. Lide, 79th ed., CRC-press, Ann Arbor 1999.

[12] N.B. Delone, V.P. Krainov, Multiphoton Processes in Atoms, 2nd ed., Springer, Berlin 2000.

[13] W.A. de Heer, Rev. Mod. Phys. 65, 611 (1995).

[14] V.P. Krainov, M.B. Smirnov, Usp. Fiz. Nauk 170, 969 (2000) [Phys. Uspekhi 43, $901(2000)]$.

[15] T. Ditmire, T. Donnelly, A.M. Rubenchik, R.W. Falcone, M.D. Perry, Phys. Rev. A 53, 3379 (1996).

[16] B.M.Smirnov, Physics of Ionized Gases, Wiley, New York 2001.

[17] E. Lefebre, G. Bonnaud, Phys. Rev. Lett. 74, 2002 (1995).

[18] E. Teller, Fusion, Academic Press, New York 1981.

[19] P.B. Parks, T.E. Cowan, R.B. Stephens, E.M. Campbell, Phys. Rev. A 63, 063203 (2001)

[20] I. Last, J. Jortner, Phys. Rev. Lett., to be published.

[21] C.D. Decker, R.A. London, Phys. Rev. A 57, 1395 (1998). 